Erratum

\title{
Binary forms and orders of algebraic number fields
}

\section{J. Nakagawa}

Joetsu University, Department of Mathematics, Joetsu, 943-Japan

Invent. Math. 97, 219-235 (1989)

There is the following serious mistake in the proof of Theorem 3 :

The estimate of Lemma 3.1 is applicable only when $X^{1 /(2 n-2)} \gg p$. Hence if $p \geqq X^{1 /(2 n-2)}$, the estimate (5.19) on p. 234 does not follow from Lemma 3.1.

The author could not recover the proof of it. Consequently, Theorems 3 and 4 are not proved. There is the following typographical mistake:

The inequality $\geqq$ on p. $229,1.16$, should be replaced by $\leqq$. 